\title{
Sickle cell disease incidence among newborns in New York State by maternal race/ethnicity and nativity
}

\author{
Ying Wang, PhD, MPH ${ }^{1,2}$, Joseph Kennedy, $\mathrm{MPH}^{3}$, Michele Caggana, ScD ${ }^{4,5}$, Regina Zimmerman, $\mathrm{PhD}^{3}$, \\ Sanil Thomas, MS', John Berninger, BS ${ }^{5}$, Katharine Harris, MBA ${ }^{5}$, Nancy S. Green, $\mathrm{MD}^{6}$, \\ Suzette Oyeku, MD, $\mathrm{MPH}^{7}$, Mary Hulihan, $\mathrm{MPH}^{8}$, Althea M. Grant, $\mathrm{PhD}^{8}$ and Scott D Grosse, $\mathrm{PhD}^{8}$
}

Purpose: Sickle cell disease is estimated to occur in 1:300-400 African-American births, with higher rates among immigrants from Africa and the Caribbean, and is less common among Hispanic births. This study determined sickle cell disease incidence among New York State newborns stratified by maternal race/ethnicity and nativity.

Methods: Newborns with confirmed sickle cell disease born to New York State residents were identified by the New York State newborn screening program for the years 2000-2008 and matched to birth records to obtain birth and maternal information. Annual incidence rates were computed and bivariate analyses were conducted to examine associations with maternal race/ethnicity and nativity.

Results: From 2000 to 2008, 1,911 New York State newborns were diagnosed with sickle cell disease and matched to the birth certificate files. One in every 1,146 live births was diagnosed with sickle cell disease. Newborns of non-Hispanic black mothers accounted for $86 \%$ of sickle cell disease cases whereas newborns of Hispanic mothers accounted for $12 \%$ of cases. The estimated incidence was 1:230 live births for non-Hispanic black mothers, 1:2,320 births for Hispanic mothers, and 1:41,647 births for non-Hispanic white mothers. Newborns of foreign-born non-Hispanic black mothers had a twofold higher incidence of sickle cell disease than those born to US-born non-Hispanic black mothers $(P<0.001)$.

Conclusion: This study provides the first US estimates of sickle cell disease incidence by maternal nativity. Women born outside the United States account for the majority of children with sickle cell disease born in New York State. Such findings identify at-risk populations and inform outreach activities that promote ongoing, highquality medical management to affected children.

Genet Med 2013:15(3):222-228

Key Words: ethnicity; foreign-born; incidence rate; maternal race; nativity; newborn screening; records linkage; sickle cell disease; US-born

\section{INTRODUCTION}

Sickle cell disease (SCD) is an inherited blood disorder causing lifelong anemia and multiorgan complications that contribute to premature death. SCD is among the most prevalent hereditary disorders in humans. It is estimated that more than 3 million Americans are genetic carriers of SCD and that 80,000-100,000 Americans have SCD. ${ }^{1-3}$ Four SCD variants-sickle cell anemia (HbSS), sickle-hemoglobin C disease (HbSC), and two types of sickle $\beta$-thalassemia ( $S \beta^{+}$thalassemia and $S \beta^{0}$ thalassemia) account for most cases of SCD.

SCD is most common among people whose ancestors came from sub-Saharan Africa; SCD is also found in India and parts of the Middle East and to a lesser extent in other Mediterranean countries. Consequently, African-Americans are at increased risk for SCD. The average frequency of SCD among AfricanAmerican births is $\sim 1$ in 360 live births and is substantially lower among US Hispanics, 1 in 16,300 live births based on national newborn screening (NBS) records pooled from states reporting such data. ${ }^{3}$ Studies estimating incidence or birth prevalence of SCD stratified by demographic characteristics such as race/ethnicity have been conducted using NBS data. ${ }^{3-6}$ However, no published information on the frequency of SCD among US births stratified by both race and ethnicity distinguishes children of US-born and foreign-born parents. The primary objectives of this study were to estimate the incidence of SCD among New York State (NYS) newborns classified by maternal race/ethnicity and nativity and to document the contribution of immigration to SCD among newborns in NYS.

\section{SCD detection \\ MATERIALS AND METHODS}

SCDs are routinely screened for and detected by the NBS Program ${ }^{7}$ in the Wadsworth Center of the NYS Department of Health. Approximately 250 SCD cases are diagnosed among $\sim 250,000$ NYS newborns each year. High-performance liquid chromatography and isoelectric focusing were used to detect

\footnotetext{
${ }^{1}$ The Congenital Malformations Registry, Bureau of Environmental and Occupational Epidemiology, Center for Environmental Health, New York State Department of Health, Albany, New York, USA; ${ }^{2}$ School of Public Health, Department of Environmental Health Sciences, University at Albany, State University of New York, Albany, New York, USA; ${ }^{3}$ Bureau of Vital Statistics, New York City Department of Health and Mental Hygiene, New York, New York, USA; ${ }^{4}$ School of Public Health, Department of Biomedical Sciences, University at Albany, State University of New York, Albany, New York, USA; ${ }^{5}$ Newborn Screening Program, Wadsworth Center, New York State Department of Health, Albany, New York, USA; ${ }^{6}$ Irving Institute Clinical and Translational Research, Division of Hematology, Columbia University Medical Center, New York, New York, USA; ${ }^{7}$ Albert Einstein College of Medicine at Yeshiva University, Children's Hospital at Montefiore, Bronx, New York, USA; ${ }^{8}$ Division of Blood Disorders, National Center on Birth Defects and Developmental Disabilities, Centers for Disease Control and Prevention, Atlanta, Georgia, USA. Correspondence: Ying Wang (wxy01@health.state.ny.us)
} 
the presence of abnormal hemoglobins (Hbs) in whole blood. High-performance liquid chromatography and isoelectric focusing are considered proven, reliable, and accurate methods for defining an infant's SCD phenotype, including HbSC, $\mathrm{HbSS}$, sickle $\beta$-thalassemia ( $\mathrm{Hb}-\mathrm{S} \beta^{+}$thalassemia and $\mathrm{Hb}-\mathrm{S} \beta^{0}$ thalassemia), and other less common forms of SCD. However, these methodologies are unable to distinguish HbSS from $\mathrm{Hb}-\mathrm{S} \beta^{0}$ thalassemia as in both cases, the result is fetal and sickle hemoglobin only. Currently, the NYS NBS Program does not perform DNA testing to distinguish $\mathrm{HbSS}$ from $\mathrm{S} \beta^{0}$ thalassemia. Therefore, the SCD subtype, HbSS, mentioned in this study also includes cases of $\mathrm{Hb}-\mathrm{S} \beta^{0}$ thalassemia.

\section{Vital records}

Live birth records of NYS residents were obtained from the Bureau of Biometrics and Health Statistics of the NYS Department of Health and the Office of Vital Statistics of the New York City Department of Health and Mental Hygiene. Access to individual level birth records containing identifying data elements for data linkage, such as name and date of birth, was approved by the NYS Department of Health Institutional Review Board (IRB) for newborns of NYS residents excluding New York City (NYC) and by the Department of Health and Mental Hygiene IRB for newborns of NYC residents.

Birth certificates were the source of information on maternal race (white, black, Asian/Pacific Islander, or other/unknown), ethnicity (Hispanic or non-Hispanic), and nativity. All subjects were categorized as white non-Hispanic, black non-Hispanic, Hispanic (regardless of race), or other. Mother's place of birth as reported in the vital record was categorized by country of birth. Puerto Rico, which is a US Commonwealth, was treated as a separate country for the purpose of this analysis. Because a high percentage $(\sim 15 \%)$ of vital records lacked father's birth country, only mother's birthplace was used in data analysis for this study. The birth records also contain birth and maternal risk factors collected in the birth certificates such as birth weight, gestational age, plurality, maternal age and education, and health insurance information.

\section{Study cohort}

Infants born during 2000-2008 with confirmed SCD were identified from the NBS Program database. A dataset containing identifying information of the SCD cases for selected birth years and the confirmatory diagnoses was prepared by NBS Program staff.

\section{Data linkage}

The study cohort was matched to the birth certificate files to obtain maternal race/ethnicity, nativity, and other demographic information of the children. As described elsewhere, ${ }^{8}$ deterministic data linkage methods widely used by public health researchers ${ }^{9-11}$ were applied using multiple criteria for establishing matches between records. As there was no unique identifier available among the two data sources, personal identifiers such as last name, sex, and date of birth were used as common identifiers in matching databases. Other potential indicators such as residential address, medical record number, and birth weight were also used to further improve the accuracy of record linkage. Several combinations of the matching variables were used to identify both definite matches and possible matches, defined as cases matched on most but not all matching variables. For the possible matches, staff manually reviewed each record to determine whether a discrepancy might be due to typographical errors or slight differences in variables such as name or address and decided whether to classify it as a match.

\section{Statistical analysis}

Annual incidence rates were computed by SCD type and by maternal and infant characteristics including race/ethnicity and nativity, birth year period, geographic location (NYC and NYS excluding NYC), infant sex, birth weight, gestational age, plurality, and maternal age. The number of SCD cases diagnosed among all NYS newborns during the study period (2000-2008) was used as the denominator and the number of live births to NYS residents during the same period was the numerator. Determination of the statistical significance of differences between groups was made using $\chi^{2}$ analysis. The data matching and the statistical analyses were performed using the Statistical Analysis System software package (SAS Institute, Cary, NC).

\section{RESULTS}

From 2000 to 2008, a total of 1,916 newborns were diagnosed with SCD among 2.2 million NYS live births or 1 in 1,146 live births during this time period. Of these identified newborns, 1,911 (99.7\%) were matched to birth certificates. Only matched SCD cases were included in the study cohort. The SCD incidence rates stratified by maternal race/ethnicity, nativity, residence, and birth period, and other selected birth and maternal risk factors are presented in Table 1.

Only minimal changes in overall SCD incidence were observed over this time period. A $1 \%$ decrease in SCD incidence among all NYS newborns was observed, from 1 in 1,115 in 2000-2002 to 1 in 1,128 in 2006-2008. A 2\% increase in incidence was observed among newborns of US-born mothers, from 1 in 1,693 to 1 in 1,664, offset by an $8 \%$ decrease in incidence among newborns of foreign-born mothers, from 1 in 655 during 2000-2002 to 1 in 714 during 2006-2008.

An important predictor of SCD incidence is maternal race/ ethnicity (Table 1). Newborns of non-Hispanic black mothers accounted for $86.0 \%$ of all SCD cases, with an average rate of 1 in 230 births. Hispanic mothers accounted for $11.5 \%$ of all newborns with SCD, for an average incidence of 1 in 2,583. About $2.5 \%$ of newborns with SCD had non-Hispanic white mothers or mothers of other maternal race/ethnicity groups. NYC accounted for $69.3 \%$ of all newborns with SCD, with an incidence of 1 in 779 live births, as compared with an average incidence of 1 in 1,975 live births in the rest of NYS.

Within every race/ethnicity group, the incidence of SCD was higher among newborns with foreign-born vs. US-born mothers (Table 1). These differences were statistically significant for 
Table 1 Numbers of live births, cases of newborns with SCD identified via Newborn Screening Program and incidence rates of SCD by demographic characteristics and maternal nativity (New York State live birth cohort, 2000-2008)



SCD, sickle cell disease.

ancludes births to mothers of unknown birth countries. bPuerto Rico, which is a US Commonwealth, was treated as a separate country for the purpose of this analysis. 'One in number of live births. ${ }^{\text {RRow percentage. }}$ Records with unknown values were not included.

Hispanics and non-Hispanic blacks and whites $(P<0.05)$. The overall annual incidence of SCD was 1 in 1,678 newborns of US-born mothers and 1 in 716 newborns of foreign-born mothers. More than half $(55.2 \%)$ of the 1,911 newborns with SCD in NYS had foreign-born mothers, in comparison with 34.5\% of all births in NYS. Nativity varied by race/ethnicity, with foreign-born mothers accounting for $12.2 \%$ of non-Hispanic white, $37.6 \%$ of non-Hispanic black, $58.0 \%$ of Hispanic, and $91.9 \%$ of Asian/Pacific Islander births in NYS.

The predominance of foreign-born mothers among NYS newborns with SCD was specific to NYC births; $60.5 \%$ of 1,325 newborns with SCD in NYC had foreign-born mothers. In comparison, $43.2 \%$ of 586 newborns born in NYS excluding NYC had foreign-born mothers. Of 1,055 newborns with SCD 
of foreign-born mothers, 802 (76\%) were born in NYC and 253 (24\%) were born in the rest of NYS.

Differences in SCD incidence by maternal nativity were found within each maternal race/ethnic group. The largest proportional difference in rates was found among non-Hispanic black mothers; the estimated annual incidence rate was 1 in 315 newborns of US-born non-Hispanic black mothers and 1 in 160 newborns of foreign-born non-Hispanic black mothers, a twofold higher rate among infants born to foreignborn non-Hispanic black mothers $(P<0.001)$. Among infants born to Hispanic mothers, the rate was 1 in 2,320 for foreignborn mothers vs. 1 in 3,173 for US-born mothers $(P=0.03)$ (Table 1). Finally, the six Asian/Pacific infants with SCD were born solely to mothers born outside the United States, resulting in an incidence of 1 in 27,673 live births.

For other birth and maternal variables examined, higher SCD incidence was observed among female children, children with low birth weight $(<2,500 \mathrm{~g})$, pre-term births ( $<37$ weeks), and children of teenage mothers (Table 1), as compared with male children, children with higher weight $(\geq 2500 \mathrm{~g})$, full-term births ( $\geq 37$ weeks), and children of older mothers ( $\geq 20$ years), respectively. The incidence of SCD was higher among newborns with foreign-born vs. US-born mothers within each variable group examined.

Table 2 shows the numbers, proportions, and incidence rates of SCD newborns (overall and by subtype) born to foreign-born non-Hispanic black mothers by maternal country of birth among the 20 countries with the largest numbers of SCD-affected births recorded. The largest absolute numbers of infants with SCD born to foreign-born non-Hispanic black mothers were seen among women born in Jamaica, followed by Haiti, Ghana, and Nigeria. However, the highest rates of SCD relative to numbers of births were seen among mothers from West Africa, including specifically Togo (1 in 43), Sierra Leone ( 1 in 50), Ghana (1 in 65), Nigeria (1 in 69), Guinea (1 in 84), and Mali (1 in 88) (Table 2). Lower SCD rates were observed among births in non-Hispanic black foreign-born mothers from the Caribbean region, including specifically Barbados (1 in 157), Antigua and Barbuda (1 in 162), Jamaica (1 in 180), and Haiti (1 in 187) (Table 2). The differences among Caribbean

Table 2 Numbers of live births, cases of SCD, and incidence rates among children of foreign-born non-Hispanic black mothers by maternal country of birth and SCD subtype for the 20 countries with the largest numbers of SCD cases recorded (New York State live birth cohort, 2000-2008)

\begin{tabular}{|c|c|c|c|c|c|c|c|}
\hline \multirow[b]{2}{*}{ Mother's birth country } & \multirow{2}{*}{$\begin{array}{c}\text { Live births } \\
n(\%)\end{array}$} & \multicolumn{2}{|c|}{ All SCDa } & \multicolumn{2}{|c|}{$\mathrm{HbSS}^{\mathrm{b}}$} & \multicolumn{2}{|c|}{$\mathrm{HbSC}$} \\
\hline & & $n(\%)$ & Rate $^{c}$ & $n(\%)^{d}$ & Rate $^{c}$ & $n(\%)^{\mathrm{d}}$ & Rate $^{c}$ \\
\hline Jamaica & $36,718(25.8)$ & 204 (22.9) & 180 & $109(53.4)$ & 337 & $88(43.1)$ & 417 \\
\hline Ghana & $6,548(4.6)$ & $100(11.2)$ & 65 & $41(41.0)$ & 160 & $58(58.0)$ & 113 \\
\hline Nigeria & $6,450(4.5)$ & $94(10.6)$ & 69 & $73(77.7)$ & 88 & $21(22.3)$ & 307 \\
\hline Guinea & $3,106(2.2)$ & $37(4.2)$ & 84 & $28(75.7)$ & 111 & $8(21.6)$ & 388 \\
\hline Mali & $2,203(1.5)$ & $25(2.8)$ & 88 & $18(72.0)$ & 122 & $5(20.0)$ & 441 \\
\hline Ivory Coast & $1,888(1.3)$ & $19(2.1)$ & 99 & $11(57.9)$ & 172 & $6(31.6)$ & 315 \\
\hline Sierra Leone & $952(0.7)$ & $19(2.1)$ & 50 & $15(78.9)$ & 63 & $4(21.1)$ & 238 \\
\hline Togo & $652(0.5)$ & $15(1.7)$ & 43 & $4(26.7)$ & 163 & $11(73.3)$ & 59 \\
\hline Senegal & $2,515(1.8)$ & $12(1.3)$ & 210 & $8(66.7)$ & 314 & $4(33.3)$ & 629 \\
\hline Antigua and Barbuda & $1,948(1.4)$ & $12(1.3)$ & 162 & $8(66.7)$ & 244 & $4(33.3)$ & 487 \\
\hline Saint Vincent and the Grenadines & $2,684(1.9)$ & $12(1.3)$ & 224 & $5(41.7)$ & 537 & $6(50.0)$ & 447 \\
\hline United Kingdom & $1,638(1.2)$ & $11(1.2)$ & 149 & $7(63.6)$ & 234 & $4(36.4)$ & 410 \\
\hline Liberia & $1,322(0.9)$ & $10(1.1)$ & 132 & $10(100.0)$ & 132 & $0(0.0)$ & - \\
\hline Saint Lucia & $2,287(1.6)$ & $9(1.0)$ & 254 & $4(44.4)$ & 572 & $5(55.6)$ & 457 \\
\hline Other countries & $14,228(10.0)$ & $54(6.1)$ & 263 & $30(55.6)$ & 474 & $22(40.7)$ & 647 \\
\hline Total & $142,300(100.0)$ & $889(100.0)$ & 160 & $525(59.1)$ & 271 & $341(38.4)$ & 417 \\
\hline
\end{tabular}

HbSC, sickle-hemoglobin C disease; HbSS, sickle cell anemia; SCD, sickle cell disease.

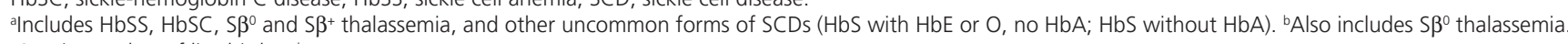




Table 3 Numbers of live births, cases of SCD, and incidence rates among children of foreign-born Hispanic mothers by maternal country of birth and SCD subtype (New York State live birth cohort, 2000-2008)

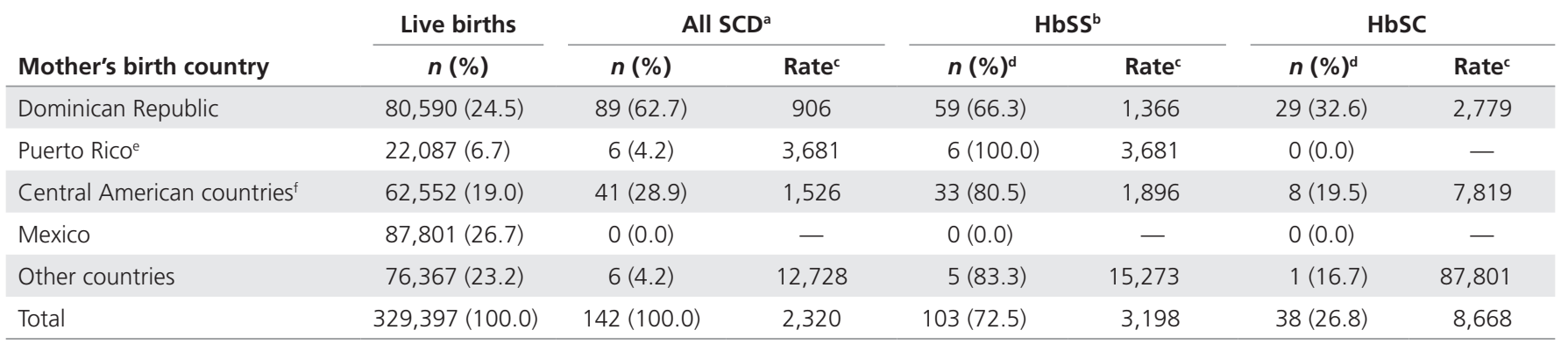

HbSC, sickle-hemoglobin C disease; HbSS, sickle cell anemia; SCD, sickle cell disease.

ancludes $\mathrm{HbSS}, \mathrm{HbSC}, S \beta^{\circ}$ and $S \beta^{+}$thalassemia, and other uncommon forms of SCD. ${ }^{b}$ Also includes $S \beta^{\circ}$ thalassemia. ${ }^{\circ}$ One in number of live births. ${ }^{\mathrm{d}}$ Row percentage.

ePuerto Rico, which is a US Commonwealth, was treated as a separate country for the purpose of this analysis. IIncludes Belize, Guatemala, Honduras, El Salvador,

Nicaragua, Costa Rica, and Panama.

countries could have been due to chance $(P=0.11)$, but the differences among African countries were statistically significant $(P<0.00001)$.

Among foreign-born mothers from West Africa, marked differences were seen in the relative frequencies of $\mathrm{HbSS}$ and $\mathrm{HbSC}$ among countries. A large majority of children with SCD born to mothers from Sierra Leone (79\%), Nigeria (78\%), Guinea (76\%), and Mali (72\%) had the HbSS phenotype; only 41 and $27 \%$ of children with SCD born to Ghanaian and Togolese mothers, respectively had HbSS (Table 2).

Table 3 summarizes the estimated annual incidence rates by country of birth and SCD subtype for foreign-born Hispanic mothers. Although the overall incidence rate in that group was 1 in 2,320, it varied for infants born to Hispanic mothers from different countries: Puerto Rico (1 in 3,681), Central American countries (Belize, Guatemala, Honduras, El Salvador, Nicaragua, Costa Rica, and Panama) (1 in 1,526), and the Dominican Republic (1 in 906). The largest number $(n=87,801)$ of foreign-born Hispanic mothers originated from Mexico. No births in this group were affected by SCD. For all other foreign-born Hispanic mothers, the overall incidence was 1 in 12,728 . Infants with SCD whose mothers were born in the Dominican Republic had a higher proportion with HbSC than other Hispanic children with foreign-born mothers (Table 3).

\section{DISCUSSION}

In this report, we present data on the incidence of SCD stratified by both maternal race/ethnicity and maternal place of birth. Previous state-based estimates of SCD incidence among newborn infants of African-American and Hispanic mothers were 1 in 396 and 1 in 36,000, respectively, in California ${ }^{5}$ and 1 in 334 and 1 in 34,500, respectively, in Texas. ${ }^{4}$ Hassell ${ }^{3}$ published a tabulation of information taken from the National Newborn Screening Information System for the years 2005-2007, according to which the average incidence of SCD was 1 in 365 births among children classified as black or African-American and 1 in 16,305 for Hispanic children. Our data show that the incidence of SCD is much higher among infants born to nonHispanic black or Hispanic mothers in New York, 1 in 230 and 1 in 2,583, respectively. Among NYS infants of US-born non-Hispanic black mothers, the rate of live births affected by SCD, 1 in 315, was at the low end of the range of previous reports cited above. The relatively high rate of SCD among NYS infants of US-born non-Hispanic black mothers could reflect an influence of the previous generation of immigrants, but we were not able to determine nativity of grandparents. The lower rates of SCD among non-Hispanic blacks in the United States relative to countries in West or Central Africa or the Caribbean could reflect a number of factors, including degree of European admixture and lesser degree of selective pressure by malaria in the United States over the course of multiple generations.

The incidence of SCD among Hispanics living in the northeastern United States is substantially higher than that among Hispanics living elsewhere in the United States, reflecting geographic differences in origins. In much of the country, Hispanics have primarily Mexican ancestry, with relatively little African ancestry or admixture. In contrast, a large proportion of Hispanics in the Northeast have Caribbean origins, especially Puerto Rico and the Dominican Republic, and many either report black race or have African admixture. An oftencited 1993 estimate of the incidence of SCD among Hispanics in the Eastern United States, including New York, was 1:1,114. ${ }^{12}$ It should be noted that that estimate was not based on NBS data, unlike our finding of a rate of 1 in 2,583 for infants of Hispanic mothers in New York. The latter is half the rate of 1 in 1,243 recently reported for Hispanics in northern Manhattan, where most are of Dominican descent. ${ }^{13}$ However, the latter estimate is close to the estimate reported in this study for Hispanic mothers from the Dominican Republic. As shown in Table 3, the incidence of SCD among Hispanics in New York varies greatly by country of origin.

The overall incidence of SCD among infants of foreign-born non-Hispanic black women in New York was 1 in 160 live births. This is comparable with the rates reported from screening programs in other countries with large immigrant populations from the Caribbean and West Africa. ${ }^{14,15}$ The incidence of SCD among NYS births to foreign-born non-Hispanic black mothers varies by country of origin: 1 in 65-70 infants born to 
mothers from Ghana or Nigeria and 1 in 180-190 infants born to mothers from Jamaica or Haiti. These estimates are consistent with data from maternal countries of origin. In Ghana, a screening study reported that 1 in 52 infants had probable SCD. ${ }^{16}$ Studies from Jamaica have reported the birth rate of SCD to be roughly 1 in 170 live births. ${ }^{17,18}$

The striking finding that $55 \%$ of all infants with SCD born in NYS during 2000-2008 were born to foreign-born mothers has important implications for US NBS programs and hemoglobinopathy centers, particularly those located in major metropolitan areas. Among live births in NYC, $61 \%$ of all infants with SCD had foreign-born mothers. Public health needs to target outreach efforts to those at-risk communities with relatively high incidences of SCD to identify their medical and social needs. Primary care and obstetrics providers can also use these data to inform their approaches to screening for SCD among immigrant children and women initially presenting for medical services.

The relative proportions of HbSS and HbSC among newborns with SCD vary by maternal country of birth. In particular, the $\mathrm{Hb}-\mathrm{C}$ allele is most frequent in Burkina Faso, Ghana, and Togo, and in those countries HbSC is more common than HbSS, unlike in most of Africa, where HbSS is the predominant form of SCD. ${ }^{19}$ The finding from our study that the majority of infants with SCD born to mothers from Ghana or Togo had HbSC is consistent with information reported previously. ${ }^{19}$ The relatively low frequency of $\mathrm{HbSC}(<20 \%)$ among infants with SCD born to foreign-born Hispanic mothers, with the exception of those from the Dominican Republic, appears to be a novel finding.

This study illustrates the value of linking multiple maternal and child health databases using individual-level linking variables. ${ }^{20,21}$ Without linking vital records to NBS records, it would not have been possible to ascertain the effect of maternal nativity on SCD incidence rates; this is the first US study to conduct such a linked analysis.

Limitations of the study include missing or incomplete information on the origins of fathers in vital records. Because this information was missing for $15 \%$ of cases, analysis was restricted to maternal country of birth. The implication of only including origin of one parent is likely attenuation of the association of parental origins with SCD incidence. However, the degree of misclassification bias appears to be low. For example, this study reported that SCD incidence among infants whose mothers were born in Jamaica was 1 in 180, whereas screening information from Jamaica indicates the incidence to be 1 in 170 births. ${ }^{17,18}$ We were not able to examine the effect of marital status on SCD incidence because that information is not collected on the NYS birth certificate. Another limitation is the ambiguity of recording race/ethnicity for people with mixed ancestries. For example, an investigation of six reported cases of SCD among infants born to foreign-born Asian/ Pacific Islander mothers (Table 1) identified mothers born in Caribbean countries who were reported to be of Asian race and Chinese ethnicity. Individuals of mixed black and Asian ancestry might have chosen to report Asian race/ethnicity. In addition, clerical errors in recording race/ethnicity are a potential limitation.

Another limitation is that SCD incidence may be underestimated due to our inability to verify the completeness of screening of all newborns in NYS. Because we did not conduct data linkage of all children in the NBS database born in the study period (2000-2008) to birth certificate records, no information about the completeness of screening was available. It was possible that the completeness of screening was $<100 \%$. This may lead to a decreased number of SCD cases being identified through NBS. However, this is unlikely to be quantitatively important. A recent study of linked Michigan birth and NBS records during January 2007 to March 2008 found that 99.2\% births were successfully matched to NBS records. ${ }^{22}$ The matching rate increased to $99.6 \%$ following a transition to a Web-based electronic birth certificate system and inclusion of a NBS card identification number on the birth record in 2008. The NYS NBS Program is currently developing a data linkage program for matching children in the NBS database to birth records to estimate the completeness of screening and identify unscreened children.

This study provides the first estimates of NYS annual SCD incidence by maternal race/ethnicity and nativity. It has been known for decades that black infants born in the Caribbean or West or Central Africa had much higher rates of SCD than black infants born in the United States due to higher frequencies of the $\mathrm{HbS}$ allele. Consistent with those data, this study has demonstrated that NYS newborns of foreignborn non-Hispanic black mothers from those countries had a substantially higher incidence of SCD as compared with NYS newborns of US-born non-Hispanic black mothers. Such findings identify at-risk populations and inform public health education and community outreach activities that promote ongoing, high-quality medical management to affected children.

\section{ACKNOWLEDGMENTS}

This project was supported, in part, by the US Centers for Disease Control and Prevention and the National Heart, Lung and Blood Institute, cooperative agreement no. U50DD000722. The authors thank Christopher Johnson of the NBS Program of the NYS Department of Health for preparing the newborn screening data for this project.

\section{DISCLOSURE}

The authors declare no conflict of interest.

\section{REFERENCES}

1. American Academy of Pediatrics. Section on Hematology/Oncology Committee on Genetics. Health supervision for children with sickle cell disease. Pediatrics 2002;109:526-535

2. Brousseau DC, Panepinto JA, Nimmer M, Hoffmann RG. The number of people with sickle-cell disease in the United States: national and state estimates. Am J Hematol 2010;85:77-78.

3. Hassell KL. Population estimates of sickle cell disease in the U.S. Am J Prev Med 2010;38(suppl 4):S512-S521. 
4. Strahan JE, Canfield MA, Drummond-Borg LM, Neill SU. Ethnic and gender patterns for the five congenital disorders in Texas from 1992 through 1998. Tex Med 2002;98:80-86.

5. Lorey FW, Arnopp J, Cunningham GC. Distribution of hemoglobinopathy variants by ethnicity in a multiethnic state. Genet Epidemiol 1996;13:501-512.

6. Lerner NB, Platania BL, LaBella S. Newborn sickle cell screening in a region of Western New York State. J Pediatr 2009;154:121-125.

7. Newborn Screening Program, New York State Department of Health. Annual Reports. Online publications. http://www.wadsworth.org/newborn/annualrept/ annsum. htm. Accessed 2 April 2012.

8. Wang $Y$, Caggana M, Sango-Jordan M, Sun M, Druschel CM. Long-term follow-up of children with confirmed newborn screening disorders using record linkage. Genet Med 2011;13:881-886.

9. Pacheco AG, Saraceni V, Tuboi SH, et al. Validation of a hierarchical deterministic record-linkage algorithm using data from 2 different cohorts of human immunodeficiency virus-infected persons and mortality databases in Brazil. Am J Epidemio/ 2008;168: 1326-1332.

10. Herman AA, McCarthy BJ, Bakewell JM, et al. Data linkage methods used in maternally-linked birth and infant death surveillance data sets from the United States (Georgia, Missouri, Utah and Washington State), Israel, Norway, Scotland and Western Australia. Paediatr Perinat Epidemiol 1997;11(suppl 1):5-22.

11. Li B, Quan H, Fong A, Lu M. Assessing record linkage between health care and Vital Statistics databases using deterministic methods. BMC Health Serv Res 2006;6:48.

12. Sickle Cell Disease Guideline Panel. Sickle cell disease: screening, diagnosis, management, and counseling in newborns and infants. Clinical Practice Guideline No. 6. AHCPR Pub. No. 93 0562. Rockville, MD: Agency for Health Care Policy and Research, Public Health Service, US Department of Health and Human Services, 1993.
13. Siddiqui S, Schunk K, Batista M, et al. Awareness of sickle cell among people of reproductive age: Dominicans and African Americans in northern Manhattan. J Urban Health 2012;89:53-58.

14. Robitaille N, Delvin EE, Hume HA. Newborn screening for sickle cell disease: A 1988-2003 Quebec experience. Paediatr Child Health 2006;11:223-227.

15. Streetly A, Latinovic R, Henthorn J. Positive screening and carrier results for the England-wide universal newborn sickle cell screening programme by ethnicity and area for 2005-07. J Clin Pathol 2010;63:626629.

16. Ohene-Frempong $\mathrm{K}$, Oduro J, Tetteh $\mathrm{H}$, Nkrumah F. Screening newborns for sickle cell disease in Ghana. Pediatrics 2008;121:S120-S121.

17. Serjeant GR, Serjeant BE, Forbes M, Hayes RJ, Higgs DR, Lehmann $H$. Haemoglobin gene frequencies in the Jamaican population: a study in 100,000 newborns. Br J Haematol 1986;64:253-262.

18. King L, Fraser R, Forbes M, Grindley M, Ali S, Reid M. Newborn sickle cell disease screening: the Jamaican experience (1995-2006). J Med Screen 2007;14:117-122.

19. Kreuels $B$, Kreuzberg $C$, Kobbe $R$, et al. Differing effects of $\mathrm{HbS}$ and $\mathrm{HbC}$ traits on uncomplicated falciparum malaria, anemia, and child growth. Blood 2010;115:4551-4558.

20. Grosse SD, Boulet SL, Amendah DD, Oyeku SO. Administrative data sets and health services research on hemoglobinopathies: a review of the literature. Am J Prev Med 2010;38(suppl 4):S557-S567.

21. Grigorescu V, Kleyn MJ, Korzeniewski SJ, Young WI, Whitten-Shurney W. Newborn screening follow-up within the lifespan context: Michigan's experience. Am J Prev Med 2010;38(suppl 4):S522-S527.

22. Korzeniewski SJ, Grigorescu V, Copeland G, et al. Methodological innovations in data gathering: newborn screening linkage with live births records, Michigan, 1/2007-3/2008. Matern Child Health J 2010;14:360-364. 